SISTEMA
ELETRÔNICO
DE REVISTAS
SER I UFPR

\title{
Determinación de los intervalos de confort y estrés térmico para espacios abiertos en la ciudad de Maringá (PR), Brasil, utilizando el índice PET
}

\section{Determinação das faixas de conforto e desconforto térmico para espaços abertos na cidade de Maringá (PR), utilizando o índice PET}

\section{Determination of thermal comfort and discomfort intervals for open spaces in Maringá city, Paraná State, Brazil, using PET index}

\author{
Mirna Milena AQUINO ${ }^{1 *}$, Paulo Fernando SOARES ${ }^{1}$, Carolina Lotufo BUENO-BARTOLOMEI ${ }^{2}$ \\ ${ }^{1}$ Universidade Estadual de Maringá (UEM), Maringá, PR, Brasil. \\ ${ }^{2}$ Universidade Estadual de Campinas (UNICAMP), Campinas, SP, Brasil. \\ *E-mail de contacto: mmilena4@gmail.com
}

Artículo recibido el 24 de julio, 2018, versión final aceptada el 24 de abril, 2019.

RESUMEN: Los modelos de predicción de confort térmico en espacios abiertos son considerados una herramienta fundamental para la planificación de nuevas urbanizaciones, así como para nuevas incorporaciones o modificaciones dentro de las ciudades. Sin embargo, la mayoría de estos índices fueron desarrollados para climas fríos. Por este motivo es necesario realizar un análisis previo de estos índices para asegurar que los mismos son representativos a la realidad del local. El objetivo de este trabajo es analizar la sensación térmica real de la ciudad de Maringá a partir del índice de Confort térmico PET. Para ello, fueron elegidos siete puntos en el centro de la ciudad donde se realizaron los levantamientos de las variables climáticas, personales y subjetivas. La metodología utilizada comprendió: el análisis del índice de confort térmico PET y la propuesta de nuevos intervalos de niveles de estrés térmico para los índices a partir de los datos particulares de la ciudad de Maringá. Como resultado del análisis se obtuvo que este índice presenta un bajo porcentaje de aciertos y correlación comparados con los votos de sensación real de las personas por lo cual se procedió a la calibración de la misma. Finalmente, realizada la calibración, se propone los siguientes intervalos de confort y estrés térmico para la ciudad de Maringá; estrés térmico para frio, $\mathrm{Ta} \leq 19,5^{\circ} \mathrm{C}$; confort, $19,5^{\circ} \mathrm{C}<\mathrm{Ta}<30^{\circ} \mathrm{C}$ y estrés térmico para calor, $\mathrm{Ta} \geq 30^{\circ} \mathrm{C}$. Esta propuesta alcanzó un porcentaje de aciertos de $67 \%$ e una correlación con la respuesta de sensación real de las 
personas de 0,29. Este resultado es compatible con otros trabajos realizados en Brasil y es aceptable para ser utilizado como herramienta predictiva de sensación térmica real de la ciudad de Maringá, PR.

Palabras claves: confort térmico; índice de Confort; PET.

RESUMO: Os modelos preditivos de conforto térmico em espaços abertos são considerados uma ferramenta fundamental para o planejamento de novas urbanizações, bem como para novas incorporações ou modificações nas urbanizações existentes. Porém, esses índices foram desenvolvidos para climas frios, pelo qual é necessário realizar uma análise prévia desses índices para garantir que sejam representativos na realidade dos locais tropicais. O objetivo deste trabalho é analisar a sensação térmica real da cidade de Maringá a partir do índice PET. Para isso, foram escolhidos sete pontos no centro da cidade no qual foram realizados os levantamentos das variáveis climáticas, pessoais e subjetivas. A metodologia utilizada incluiu; a análise do índice de conforto térmico PET e a proposta de novos intervalos de níveis de estresse. Como resultado da análise, verificou-se que o índice apresentava baixa resposta em quanto a porcentagem de acertos e correlação comparados com os votos de sensação real das pessoas pelo qual se procedeu à calibração da mesma. Finalmente, realizada a calibração, sugerem-se as seguintes faixas de conforto/desconforto térmico: desconforto para o frio, Ta $\leq$ $19,5^{\circ} \mathrm{C}$; conforto, $19,5^{\circ} \mathrm{C}<\mathrm{Ta}<30^{\circ} \mathrm{C}$ e desconforto para o calor, $\mathrm{Ta} \geq 30^{\circ} \mathrm{C}$. A proposta apresentada uma porcentagem de acertos de $67 \%$ e uma correlação com as respostas dos votos de sensação térmica real de 0,29 . Os resultados deste trabalho são compatíveis com as calibrações anteriormente realizadas no Brasil além de, satisfatória para ser utilizado como ferramenta preditiva da sensação térmica da cidade de Maringá.

Palavras-chave: conforto térmico; índice de Conforto; PET.

ABSTRACT: Prediction models of thermal comfort in open spaces are considered a fundamental tool in neighborhood planning, as well as in open space design within urban areas. However, most of these indices were developed for cold climates. For this reason, conducting a preliminary analysis is necessary to ensure that they are suitable for a location presenting a different, warmer climate. The objective of this work is to analyze the real thermal sensation in the city of Maringá, Paraná State, Brazil, based on PET (thermal comfort index). For this, we collected information on climate, users and subjective variables at seven locations spread throughout the downtown area. The analysis allowed for the proposal of new intervals of thermal stress levels for Maringá. The index presented a low percentage of correct answers and correlation with the votes of real sensation, for this reason, calibration was required. The proposed range of thermal comfort and thermal stress for Maringá City is: cold thermal stress, $\mathrm{Ta} \leq 19.5^{\circ} \mathrm{C}$; thermal comfort, $19.5^{\circ} \mathrm{C}<\mathrm{Ta}<30^{\circ} \mathrm{C}$ and hot thermal stress, $\mathrm{Ta} \geq$ $30^{\circ} \mathrm{C}$. This proposal reached $67 \%$ of percentage of correct answers and 0.29 of correlation with the response of real thermal sensation. This result is compatible with other works conducted in Brazil and its use is acceptable as a predictor of real thermal sensation in the city of Maringá, PR.

Keywords: thermal comfort; thermal index; PET.

\section{Introducción}

En la actualidad los innumerables cambios climáticos, como consecuencia de las alteraciones realizadas por el hombre, están afectando el confort urbano (Capelli et al., 2005). La falta de confort urbano presenta repercusión directa en el uso de los espacios abiertos que tiende a reducirse (Araújo \& Rangel, 2016). Solo en los Estados Unidos, una persona permanece en espacios cerrados el $87 \%$ de su tiempo, $5 \%$ a $6 \%$ descolándose, y apenas un $8 \%$ en espacios abiertos, número que puede disminuir 
de $2 \%$ a $4 \%$ en periodos de mucho calor o frio. Conjuntamente a las características climáticas del local, las características de un ambiente urbano denso también colaboran para que las personas no duren en el exterior, reforzando la tendencia a permanecer en el interior de las edificaciones con las ventanas cerradas (Farr, 2013).

Labaki et al. (2012) afirma que, para algunas personas, la calle es sólo la senda de uso temporal, sin embargo, para otras, ella puede significar más que solo un itinerario. Entretanto, para que esto suceda, los espacios públicos deben ser dotados de mayor estructura, equipamientos, seguridad, además de una buena calidad ambiental, que incluye microclimas favorables para el confort térmico.

Con relación a la sensación térmica, el clima, es característico de cierta localidad geográfica, de modo a que no hay dos climas rigurosamente iguales (Barbirato et al., 2007), también ocurre que la adaptabilidad de las personas al clima de un local determinado difiere de un individuo a otro, esto hace que la sensación térmica sea diferente de persona a persona (Cohen et al., 2013). Resaltando lo anterior, la adaptación psicológica es de extrema importancia, puesto que los factores de conducta y psicológicos afectan la satisfacción de los usuarios y contribuye para diferencias que existen en los votos de sensación de confort calculado por medio de los índices predictivos (Labaki et al., 2012).

Sartori (2000) hace hincapié en la importancia de recolectar estos datos por medio de entrevistas a los usuarios y presenta algunas cuestiones a ser consideradas al momento de realizar estudios sobre clima y como los individuos lo perciben. Estas variables incluyen lugar de residencia y origen de la persona, edad, nivel de educación, percepción del ambiente y clima del local.
Al hablar del análisis de Índices de Confort o estrés térmico es necesario, por tanto, analizar variables climáticas, personales y subjetivas. Para la recolección de estos datos, la ISO 10551, la ISO 7726 y la ASHRAE 55 establecen parámetros de referencia utilizados en varios trabajos tanto en Brasil como en otros países (Monteiro, 2008; Krüger \& Rossi, 2011; Rossi, 2012; Cohen et al., 2013; Lin et al., 2013), los cuales apuntan a investigar diferentes índices en espacios abiertos y verificar su aplicabilidad como índice predictivo de confort térmico, así también, contribuyendo a la calibración de estos para la realidad del local analizado.

En Brasil, considerando los últimos 10 años, el trabajo de Montiero (2008) es reseña para varias investigaciones realizadas para el estado de San Pablo. Monteiro (2008) realiza la calibración de 21 modelos de predicción de confort e/o estrés térmico para espacios abiertos de la ciudad de São Paulo, SP, Brasil. Pezzuto (2007), por su vez, evalúa la sensación de confort térmico en la ciudad de Campinas, SP, Brasil, utilizando como índice predictivo el índice PMV. Bröde et al. (2011) estudian el impacto de la temperatura, la humedad, el viento y el flujo de calor radiante de ondas largas y cortas en la sensación de confort térmico, alineados con los votos de sensación térmica y comparados con las previsiones del índice UTCI. Labaki et al. (2012) presentan los resultados de un estudio sobre el confort térmico en espacios abiertos de pasaje en calles peatonales de las ciudades de Campinas, Bauru y Presidente Prudente, en el estado de São Paulo utilizando el índice PET. Abreu-Harbich et al. (2014) cuantifican las mudanzas en el confort térmico debido a las configuraciones típicas de los Canyos urbanos de Campinas, utilizando el índice PET. Decanal et al. (2010) investigan el papel de 
los fragmentos forestales urbanos en el confort térmico dentro de la ciudad de Campinas utilizando los índices PMV y PET.

De forma paralela, Rossi (2012) analiza la sensación térmica de la población de Curitiba por medio de los índices PMV, PET y UTCI. Do Couto et al. (2013) utilizan los índices PET y UTCI para identificar a qué clase de estrés térmico están sometidos los usuarios del Campus de la UFMT en Cuiabá, Brasil. Souza (2010) realiza la calibración de los índices de confort térmico PET e PMV según la percepción de los habitantes de la ciudad de Salvador/BA, Brasil, y posteriormente, la evaluación del desempeño térmico de los espacios abiertos urbanos. Hiroshima (2014) estudia la percepción sonora y térmica, y el confort acústico y térmico de los usuario de los espacios abiertos de la ciudad de Belo Horizonte/MG, además realiza la calibración de los parámetros $\mathrm{L}_{\text {Aeq, } \mathrm{T}} \mathrm{y}$ PET.

Así pues, en la literatura el índice PET (Physiological Equivalent Temperature) es considerado como uno de los principales índices analizados para determinar el confort térmico en personas. Basado en el modelo de Munich - MEMI (Höppe, 1999; Monteiro \& Alucci, 2007), representa una temperatura ficticia, resultante de la interacción entre variables físicas y ambientales de un ambiente real. Desarrollado por Mayer \& Höppe (1987) para ser aplicado tanto en ambientes externos como en ambientes internos.

De esta manera, se define como objetivo de trabajo, analizar el confort térmico de las personas de la ciudad de Maringá/PR y determinar los intervalos de confort y estrés térmico del índice PET para las condiciones climáticas locales y la sensación térmica real de los habitantes, para lo cual, fueron analizados los datos climáticos, datos personales y subjetivos de los personas obtenidos por medio del monitoreo en campo y entrevistas realizadas en las aceras de la ciudad durante los meses de verano (enero y febrero) e invierno (junio y julio).

\section{Caracterización del local de estudio}

La ciudad de Maringá fue seleccionada como local de estudio. Geográficamente Maringá se encuentra en la latitud de $23^{\circ} 25^{\prime} 31^{\prime \prime} \mathrm{S}$, longitud de 51 ${ }^{\circ} 56^{\prime} 19^{\prime \prime} \mathrm{W}$ y altitud de $596 \mathrm{~m}$ (IPARDES, 2016). De acuerdo con la clasificación de Köppen (Figura 1), el clima de Maringá es de tipo Cfa (clima subtropical), caracterizado por temperatura promedio inferior a $18^{\circ} \mathrm{C}$ (invierno) y temperaturas promedio arriba de $\operatorname{los} 22^{\circ} \mathrm{C}$ (verano), con veranos calurosos y concentración de lluvias dentro de estos meses, helada poco frecuentes y sin estación seca definida (IAPAR,2016).

La zona de estudio se concentra en el centro urbano de esta ciudad específicamente a lo largo de la Avenida Brasil que concentra el área comercial de la ciudad. Esta avenida une el centro de Maringá en el sentido este-oeste, paralela a la línea férrea (Rego, 2001), fue proyectada para cumplir el papel de eje central del comercio de la ciudad (Andrade \& Souza, 2008), compuesto por una avenida de dos carriles en ambos sentidos de circulación, cantero central con ciclovía y arboles de Ipê roxo (Lapacho) presentes dentro del corredor (Rego, 2001)

Parte de la Av. Brasil está ubicada dentro de lo que se conoce como "contorno Binario", cuyos límites son las avenidas perpendiculares Paraná y São Paulo. Dentro de estos límites fueron seleccionados siete puntos de medición donde se llevó acabo el levantamiento de las variables climáticas 


\section{Classificação Climática - Segundo Köppen}

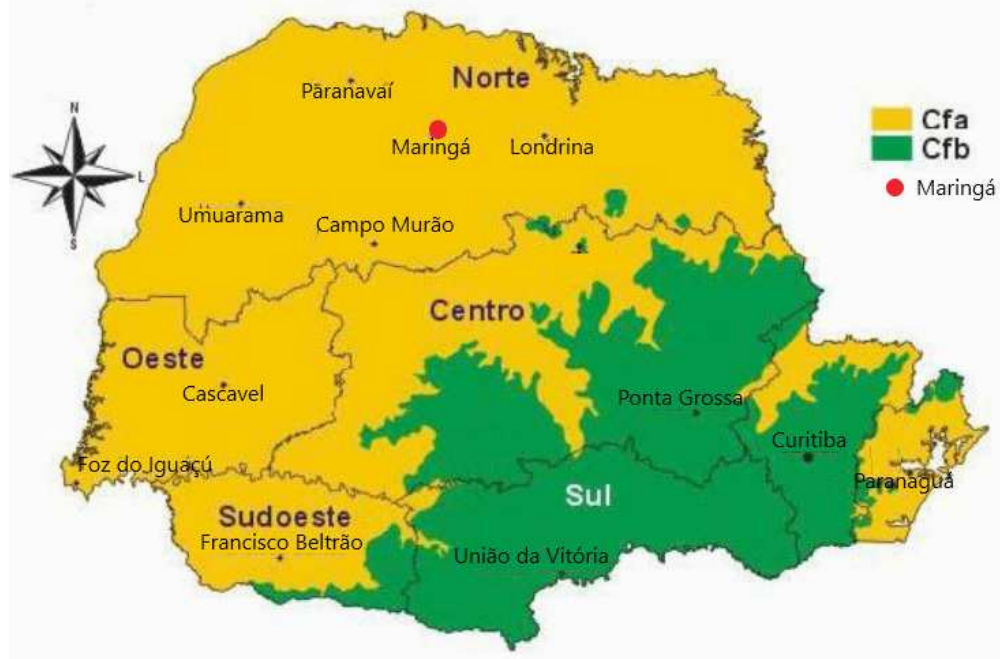

FIGURA 1 - Clasificación Climática según Köppen.

FUENTE: IAPAR, 2016.

(temperatura, humedad relativa e velocidad de los vientos), variables personales (edad, sexo, altura, vestimenta) y variables subjetivas (sensación y preferencias).

Específicamente, se seleccionaron seis intersecciones y una plaza localizadas dentro de estos límites. Las mediciones se desarrollaron de 10:00hs a 15:00hs de forma continua por siete días durante el mes de enero y catorce días en febrero, mediciones correspondientes al periodo de verano, además de, catorce días en junio y siete días en el julio, correspondientes al periodo de invierno.

Los locales mencionados fueron escogidos de acuerdo con la cantidad de personas que circulan diariamente dentro del área de influencia, así como también, teniendo en cuenta la presencia o no de conjunto de árboles, así como también, el lado de la acera. De esta manera, fueron localizados tres locales de medición del lado derecho y tres del lado izquierdo de la avenida sobre las aceras. Para el último local fue seleccionada una plaza localizada dentro el área de estudio, en el margen derecho de la avenida y aproximadamente, a mitad de cuadra (Tabla 1).

TABLA 1 - Localización de los puntos seleccionados.

\begin{tabular}{ccc}
\hline Local & Situación & Descripción \\
\hline 1 & Intersección & Av. Brasil e Av. Paraná \\
2 & Plaza & Plaza Napoleão Moreira da Silva \\
3 & Intersección & Av. Brasil e Av. Duque de Caxias \\
4 & Intersección & Av. Brasil y Av. Herval \\
5 & Intersección & Av. Brasil y Getúlio Vargas \\
6 & Intersección & Av. Brasil y Calle Piratininga \\
7 & Intersección & Av. Brasil y Av. São Paulo \\
\hline
\end{tabular}

FUENTE: Autores, 2017. 
En la Figura 2 se presenta una imagen aérea del área de estudio con la localización de los puntos con relación el Tabla 1. Con relación a las características urbanas de los locales, las avenidas y calles circundantes a los locales de medición, se encuentran asfaltadas, con veredas de materiales semejantes, edificaciones de hasta 3 pisos y vitrinas de comercios a lo largo del corredor.

\section{Materiales y métodos}

En la Tabla 2 se presenta los días y locales en los cuales fueron realizados el levantamiento de datos climáticos, personales y subjetivos según los periodos de verano e inverno.

Las variables climáticas levantadas fueron: Temperatura de aire (Ta), Temperatura de Globo (Tg), Humedad relativa (UR), Velocidad y dirección del viento (Va). Estos valores fueron medidos con ayuda del instrumento de medición Data Logger
Testo H-117 y un anemómetro térmico de precisión. En la Figura 3 se visualizan ambos dispositivos en campo, las mediciones de temperaturas y humedad fueron realizadas de manera automática cada 5 segundos utilizando el Data Logger y de forma manual, las mediciones de dirección y velocidad del viento registrándolos cada 30 minutos.

En todas las mediciones realizadas, los dispositivos estuvieron posicionados mirando a la Av. Brasil a 1,1 metros de altura con relación al suelo según recomendación de la Norma ISO 7726 (1998), sobre las veredas cercano a las edificaciones con excepción de los locales 2 y 3 que fueron posicionados en la acera de la plaza.

Las variables personales y subjetivas fueron levantadas a partir de un cuestionario pre-formulado basado en los requerimientos de la Norma Internacional ISO 10551 (1995) y el trabajo de Rossi (2012). El cuestionario fue aplicado a las personas que circulaban por el área de influencia.

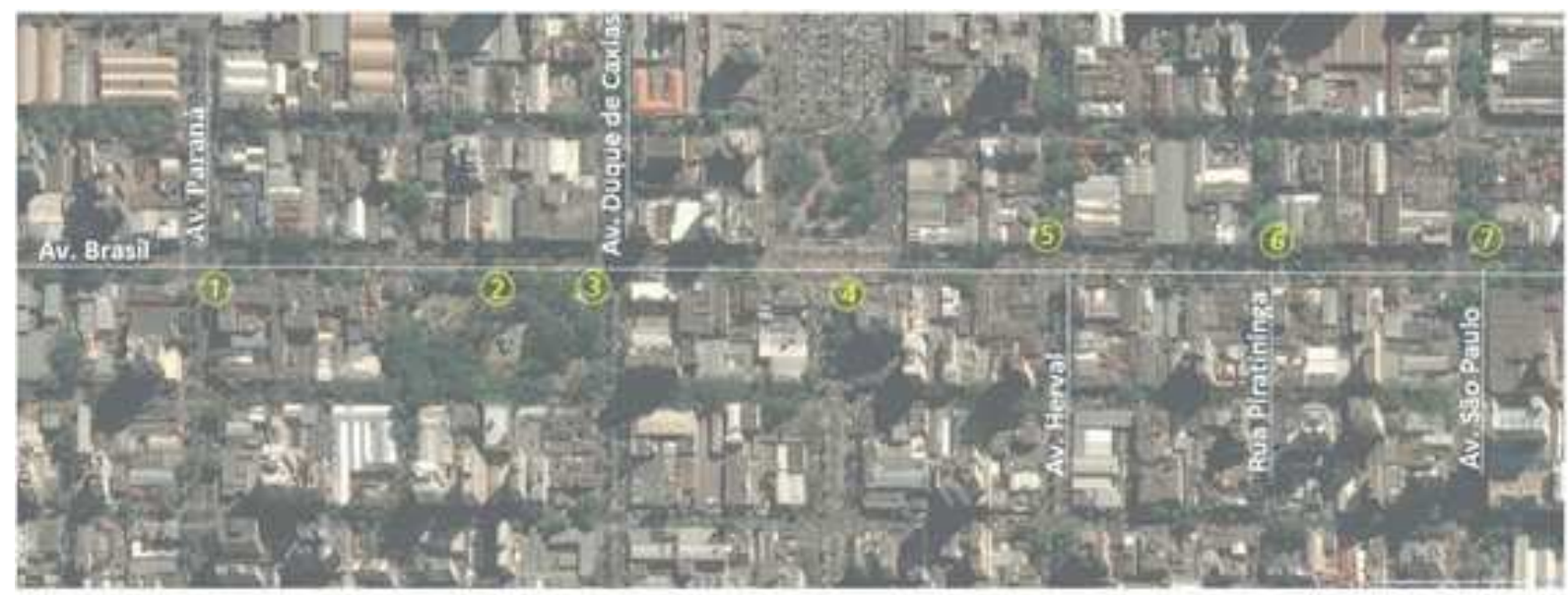

FIGURA 2 - Localización de los puntos seleccionados.

FUENTE: Mapas Bing (05/06/2017). 
Con relación a las variables personales, fue reunida información sobre: (1) género, (2) edad, (3) altura, (4) peso, (5) ciudad en la vive y tiempo de permanecía, además de (6) vestimenta y (7) tiempo de exposición al ambiente externo. Sobre las variables subjetivas, en la Figura 4 se visualiza las preguntas del cuestionario relacionado con la sensación térmica (pregunta 1), satisfacción (pregunta 2), preferencia (pregunta 3) y aceptación térmica

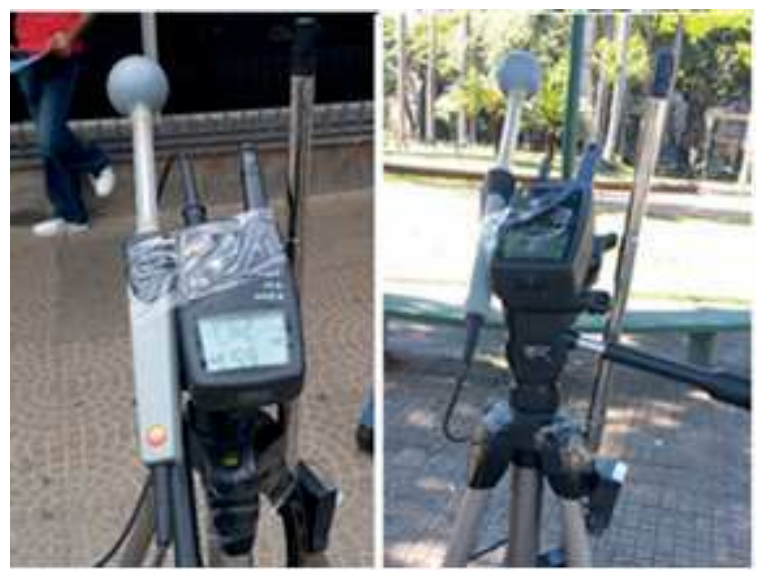

FIGURA 3 - Dispositivos utilizados para el levantamiento de los datos climáticos.

FUENTE: Autores, 2016.

TABLA 2 - Días y locales analizados por mes.

\begin{tabular}{|c|c|c|c|c|c|c|c|}
\hline Locales & Av. Paraná & $\begin{array}{l}\text { Plaza Morei- } \\
\text { ra Da Silva }\end{array}$ & $\begin{array}{l}\text { Av. Duque de } \\
\text { Caxias }\end{array}$ & $\begin{array}{l}\text { Getúlio } \\
\text { Vargas }\end{array}$ & Av. Herval & $\begin{array}{l}\text { Calle Pirati- } \\
\text { ninga }\end{array}$ & $\begin{array}{l}\text { Av. São } \\
\text { Paulo }\end{array}$ \\
\hline \multirow{3}{*}{ Verano } & 20/Ene/16 & 21/Ene/16 & 22/Ene/16 & 29/Ene/16 & 26/Ene/16 & 27/Ene/16 & 28/Ene/16 \\
\hline & $01 / \mathrm{Feb} / 16$ & $02 / \mathrm{Feb} / 16$ & 03/Feb/16 & $06 / \mathrm{Feb} / 16$ & $05 / \mathrm{Feb} / 16$ & $11 / \mathrm{Feb} / 16$ & $08 / \mathrm{Feb} / 16$ \\
\hline & $10 / \mathrm{Feb} / 16$ & 12/Feb./16 & $15 / \mathrm{Feb} / 16$ & $20 / \mathrm{Feb} / 16$ & $16 / \mathrm{Feb} / 16$ & 18/Feb/16 & 19/Feb/16 \\
\hline \multirow{3}{*}{ Invierno } & 7/Jun/16 & 08/Jun/16 & 09/Jun/16 & 13/Jun/16 & 10/Jun/16 & 16/Jun/16 & 14/Jun/16 \\
\hline & 15/Jun/16 & 17/Jun/16 & 20/Jun/16 & 24/Jun/16 & 21/Jun/16 & 22/Jun/16 & 23/Jun/16 \\
\hline & 4/Jul/16 & 05/Jul/16 & 07/Jul/16 & 12/Jul/16 & 08/Jul/16 & 09/Jul/16 & 11/Jul/16 \\
\hline
\end{tabular}

FUENTE: Autores, 2017.

de los usuarios (pregunta 4). El último punto del cuestionario, pregunta 5, fue elaborado para analizar la influencia del diseño urbanos en la sensación del confort térmico.

Para determinar el número de entrevistas representativas a ser aplicadas fue considerado la población de Maringá mayor a 15 años y calculado con ayuda de la ecuación estadística de muestra aleatoria simple. La población de Maringá mayor a 15 años es de 301596 (IPARDES, 2016), lo que dio como resultados 399 entrevistas a ser aplicadas en cada periodo (verano e invierno). Esta cantidad fue considerada para el periodo de verano e invierno, buscando distribuirlos en un mínimo de 21 entrevistas por día.

Una vez levantados los datos, estos fueron tabulados y organizados en planillas electrónicas para su posterior análisis. Siguiendo las recomendaciones de la ASHRAE Standard 55 (2004), no fueron consideradas como parte de la muestra, las personas que declararon estar al aire libre a menos de 15 minutos. Por otra parte, con relación a la ciudadanía, solo fueron considerados aquellas personas que viven dentro de Maringá y las ciudades limítrofes (Sarandi, Floresta y Paiçandu). 
Seguido a esto, fue realizado la clasificación de los datos en tres grupos: Personas Confortables (C), Personas incomodas (estrés) con tendencia al frio (DF) y Personas incomodas (estrés) con tendencia al calor (DC). Esta clasificación fue realizada llevando en cuenta la combinación de respuestas al cuestionario presentadas en el Tabla 3, la combinación de respuestas fuera de estos grupos fue descartados de los datos tabulados.

En total, se aplicaron 886 (ochocientos ochenta y seis) entrevistas, de estas, primeramente, fueron excluidas aquellas con respuesta de vivienda y tiempo de permanencia fuera de los intervalos descritos arriba, restando así 623 entrevistas. Luego, de proceder a la clasificación de las entrevistas según personas confortables y personas con estrés térmico para frio o para calor, según lo presentado en el Tabla 3, restando 351 datos a ser analizados, 151 (43\%) entrevistas en el grupo de Personas Confortables (C), 173 (49,3\%) entrevistas en el grupo con estrés para el calor (DC) y $27(7,7 \%)$ para el grupo con estrés para el frío (DF).

\section{Neste exato momento, vocé está sentindo:}

\begin{tabular}{|c|c|c|c|c|c|c|}
\hline Mulito frio & Frio & $\begin{array}{c}\text { Um pouco } \\
\text { frio }\end{array}$ & $\begin{array}{c}\text { Neen frio } \\
\text { nemicalor }\end{array}$ & $\begin{array}{c}\text { Um pouco } \\
\text { de calor }\end{array}$ & Calor & $\begin{array}{c}\text { Muito } \\
\text { Calor }\end{array}$ \\
\hline
\end{tabular}

\section{Neste exato momento, voct está:}

\begin{tabular}{|c|c|c|c|}
\hline Confartivel & $\begin{array}{c}\text { Um pouco } \\
\text { desconfortavel }\end{array}$ & Desconfortavel & $\begin{array}{c}\text { Muito } \\
\text { desconfortaivel }\end{array}$ \\
\hline
\end{tabular}

\section{Neste exato momento, voce preferiria estar sentindo:}

\begin{tabular}{|c|c|c|c|c|c|c|}
\hline $\begin{array}{c}\text { Muito } \\
\text { man Frio }\end{array}$ & Mais Frio & $\begin{array}{c}\text { Um pouce } \\
\text { mais frio }\end{array}$ & $\begin{array}{c}\text { Serm } \\
\text { mudanca }\end{array}$ & $\begin{array}{c}\text { Um pouco } \\
\text { mais de } \\
\text { celor }\end{array}$ & Mais Calor & $\begin{array}{c}\text { Muito } \\
\text { mais Calor }\end{array}$ \\
\hline
\end{tabular}

4. Levando em conta suas preferèncias pessoais, você aceitaria as condiç̄oses climúticas:

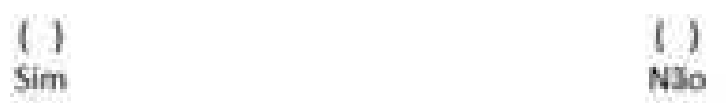

5. Neste exato momento, com relaça às condipbes climśticas, na sua opinialo estar neste local 6 :

\begin{tabular}{|c|c|c|c|}
\hline $\begin{array}{c}\text { Perfeitamente } \\
\text { toleraivel }\end{array}$ & $\begin{array}{c}\text { Facilmente } \\
\text { tolerajuel }\end{array}$ & $\begin{array}{c}\text { Dificitmente } \\
\text { tolerifvel }\end{array}$ & Intolerawel \\
\hline
\end{tabular}

FIGURA 4 - Cuestionario referente a las variables subjetivas.

FUENTE: Autores, 4/06/2017. 
TABLA 3 - Preguntas y respuestas sobre las variables Subjetivas.

\begin{tabular}{|c|c|c|c|c|}
\hline & Pregunta & Confortable (C) & Estrés para el frio (DF) & Estrés para el calor (DC) \\
\hline 2. & $\begin{array}{c}\text { En este Exacto momento, usted } \\
\text { está }\end{array}$ & Confortable & $\begin{array}{l}\text { Un poco incomodo } \\
\text { Incomodo } \\
\text { Muy Incomodo }\end{array}$ & $\begin{array}{l}\text { Un poco incomodo } \\
\text { Incomodo } \\
\text { Muy incomodo }\end{array}$ \\
\hline 3. & $\begin{array}{c}\text { En este Exacto momento, usted } \\
\text { preferiría sentir (preferencia } \\
\text { térmica) }\end{array}$ & Sin mudanza & $\begin{array}{l}\text { Mucho más Frio } \\
\text { Más Frio } \\
\text { Un poco más de frio }\end{array}$ & $\begin{array}{l}\text { Un poco más de calor } \\
\text { Más Calor } \\
\text { Mucho más calor }\end{array}$ \\
\hline 4. & $\begin{array}{l}\text { Teniendo en cuenta sus preferen- } \\
\text { cias personales, ¿usted aceptaría } \\
\text { las condiciones climáticas? }\end{array}$ & $\mathrm{Si}$ & $\mathrm{No} / \mathrm{Si}$ & $\mathrm{No} / \mathrm{Si}$ \\
\hline
\end{tabular}

FUENTE: Autores, 2017.

\section{Análisis índice PET}

El índice PET fue calculado con el programa RayMan 2.0 el cual fue desarrollado por Andreas Matzarakis. Este programa permite la inserción de datos iniciales (input) de variables climáticas: temperatura, humedad relativa del aire, velocidad del aire, y datos personales: género, edad, altura, peso, vestimenta (clo) y metabolismo (W).

Para este análisis, fue considerado como tasa metabólica igual a $165 \mathrm{~W} / \mathrm{m}^{2}$, correspondiente a una persona caminado en una superficie plana a $4 \mathrm{~km} / \mathrm{h}$. Para calcular la tasa metabólica en W de cada persona, primeramente, fueron calculados los valores de las áreas de la superficie corporal $\left(\mathrm{m}^{2}\right)$ de los entrevistados, a continuación, ese valor fue multiplicado por el valor de la tasa metabólica en $\mathrm{W} / \mathrm{m}^{2}$ para, finalmente, obtener la tasa Metabólica en Watts $(\mathrm{W})$ para cada individuo.

Una vez calculados el índice PET para cada individuo, estos valores fueron relacionados con los votos de sensación real de las personas (Tabla 4) y analizados los porcentajes de aciertos y nivel de correlación con los mismos según la siguiente clasificación:

(1) Estrés para el frio, considerados valores de Sensación térmica: un poco de frio, Frio y Mucho frio con valor de $\mathrm{PET} \leq 13^{\circ} \mathrm{C}$.

(2) Confortable, Valor de sensación térmica "ni calor ni frio" y; $13^{\circ} \mathrm{C}<\mathrm{PET}<29^{\circ} \mathrm{C}$, y

(3) Estrés para el calor, valores de sensación térmica de: calor, un poco de calor y mucho calor con valor de $\mathrm{PET} \geq 29^{\circ} \mathrm{C}$.

Esta misma clasificación fue utilizada para realizar el análisis de los valores calibrados. Los límites calibrados fueron determinados por el método interactivo, buscando maximizar el porcentaje de aciertos y el nivel de correlación del índice con los votos de sensación real. 
TABLA 4 - Escala de estrés térmico versus sensación térmica real.

\begin{tabular}{|c|c|c|}
\hline $\operatorname{PET}\left({ }^{\circ} \mathrm{C}\right)$ & $\begin{array}{l}\text { Sensación térmica } \\
\text { (ST) }\end{array}$ & Nivel de estrés térmico \\
\hline$<4$ & \multirow{2}{*}{ Mucho frio } & Extremo estres para frio \\
\hline $4-8$ & & Forte estrés para frio \\
\hline $8-13$ & Frio & Moderado estres para frio \\
\hline $13-18$ & Un Poco frio & Leve estrés para frio \\
\hline $18-23$ & Ni frio ni calor & Sin estrés térmico \\
\hline $23-29$ & Un Poco de calor & Leve estrés para calor \\
\hline $29-35$ & Calor & Moderado estrés para calor \\
\hline $35-41$ & \multirow{2}{*}{ Mucho calor } & Forte estrés para calor \\
\hline$>41$ & & Extremo estrés para calor \\
\hline
\end{tabular}

FUENTE: Autores, 2017.

\section{Resultados}

En esta sección, primeramente, serán presentados la distribución de las variables personales, subjetivas y las variables climáticas para luego iniciar con el análisis de estos datos a partir del índice PET.

Del total de entrevistas, 192 fueron aplicadas a mujeres e 132 a hombres (Figura $4-a$ ). Para la clasificación de datos según faja etaria fue realizado conforme a las Naciones Unidas (1983) en: Adolescente: menor de 14 años; Joven: de 15 a 24 años; Joven Adulto: de 25 a 44 años; adulto: de 45 a 64 años y Adulto mayor: arriba de 65 años. Es así como, el 89\% (312) de los entrevistados integran los grupos joven, joven adulto y adulto, restando solo un $11 \%$ (39) para los grupos adolescente y adulto mayor (Figura $4-\mathrm{d}$ ).

Con relación a la tasa metabólica el intervalo de valores se encuentra dentro de los 250,8 y 348,6 Watts (Figura $4-$ b) correspondientes a personas de entre 1,58 y 1,80 metros de altura y con peso entre 53 a 93 kilos. Entre tanto, los valores de resistencia térmica de la vestimenta fueron divididos en cuatro grupos; menores de 0,5 clo., entre 0,5 clo y 1,00 clo., entre 1,01 clo. y 1,50 clo. y mayores de 1,50 clo. (Figura $4-\mathrm{c}$ ). De estas 4 clasificaciones, la mayoría de las personas que afirmaron encontrarse confortables correspondían el grupo de personas con resistencia térmica de su vestimenta entre 0.5 y 1.00 clo., es de notar también que, tomando el total de datos, en cuanto a la resistencia térmica, el mayor número de entrevistados se encuentra entre valores menores de 0.5 clo., sin embargo, afirmaron encontrarse incomodos con tendencia al calor.

Por otra parte, con relación a las variables ambientales, las temperaturas medias de los meses de verano estuvieron entre $\operatorname{los} 27,3^{\circ} \mathrm{C}$ y $\operatorname{los} 33,0^{\circ} \mathrm{C}$, en la Figura 6 se observa los datos distribuidos conforme a los locales de medición divididos entre los meses de enero (1 semana) y febrero (2 semanas). Los datos Febrero 1 corresponden a los primeros 7 días de medición del mes de Febrero en los 7 puntos seleccionados, y Febrero 2, corresponde a las segundas mediciones realizadas en los puntos de medición en el mes de Febrero.

Como se puede observar en la Figura 5, las mediciones no necesariamente fueron realizadas siguiendo el orden de los locales (puntos), esto quiere decir que, entre la fecha de medición del local de la Av. Paraná (Punto 1) y del local de situado en la Plaza Moreira da Silva (Punto 2) puede haber más de dos días de diferencia. La concurrencia de lluvias durante los días de mediciones junto con eventos sociales registrados en los puntos, fueron algunas de las causantes de diferencias en los días. Respecto a los valores medidos, se obtuvieron 8 días con temperaturas medias arribas de los $30,0^{\circ} \mathrm{C}$ (líneas), 
entretanto, las máximas superaron $\operatorname{los} 40^{\circ} \mathrm{C}$ en dos días dentro de las mediciones, y estos días pueden identificarse claramente en los picos del periodo de Enero y Febrero 2.
En lo que respecta a la humedad relativa del periodo en cuestión, estas estuvieron en el rango de los $39,5 \%$ y $77,7 \%$ (barras), hubo, sin embargo, en 4 días periodos en los que fueron registrados valores
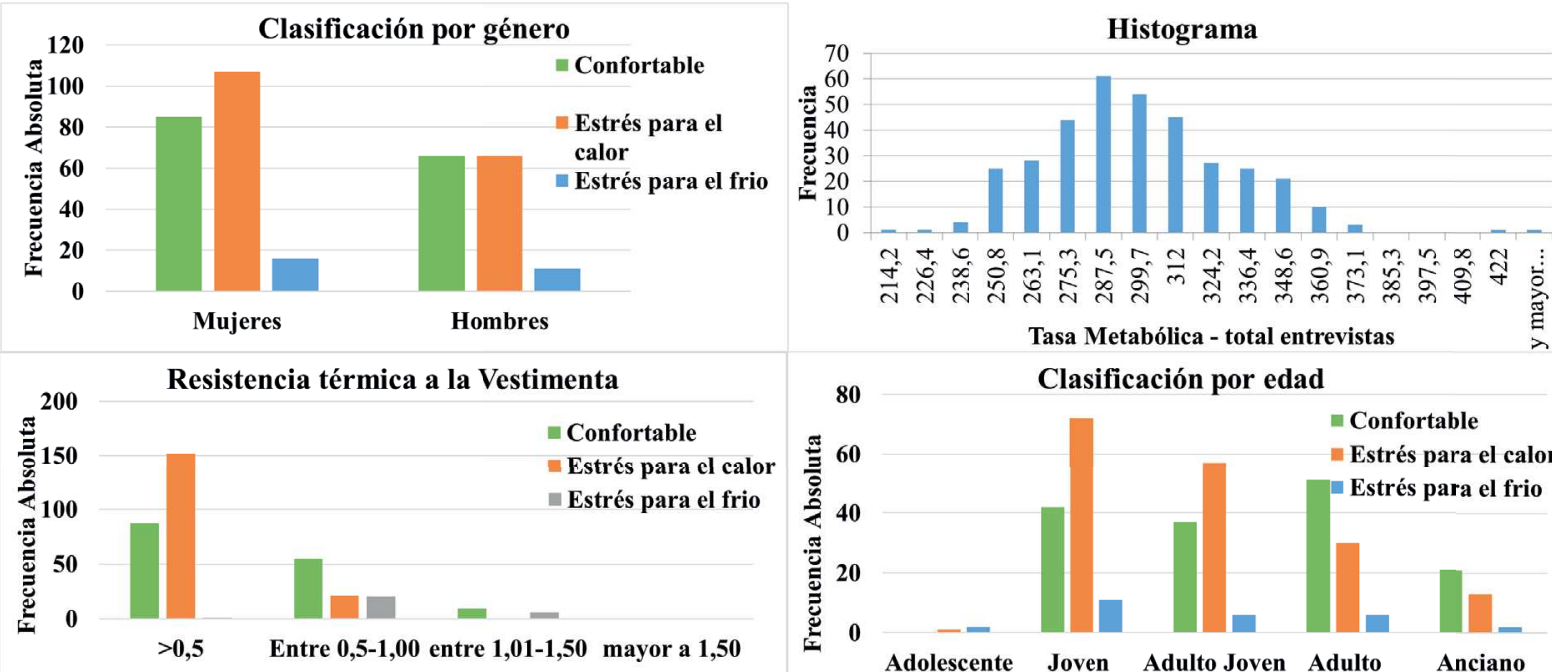

FIGURA 4 - Datos sobre las variables personales analizadas.

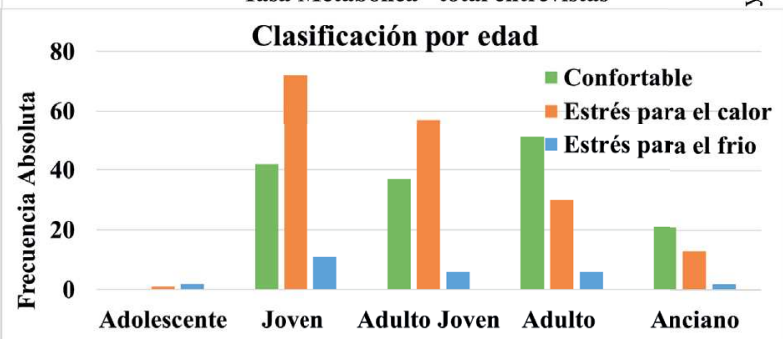

FUENTE: Autores, 2017.

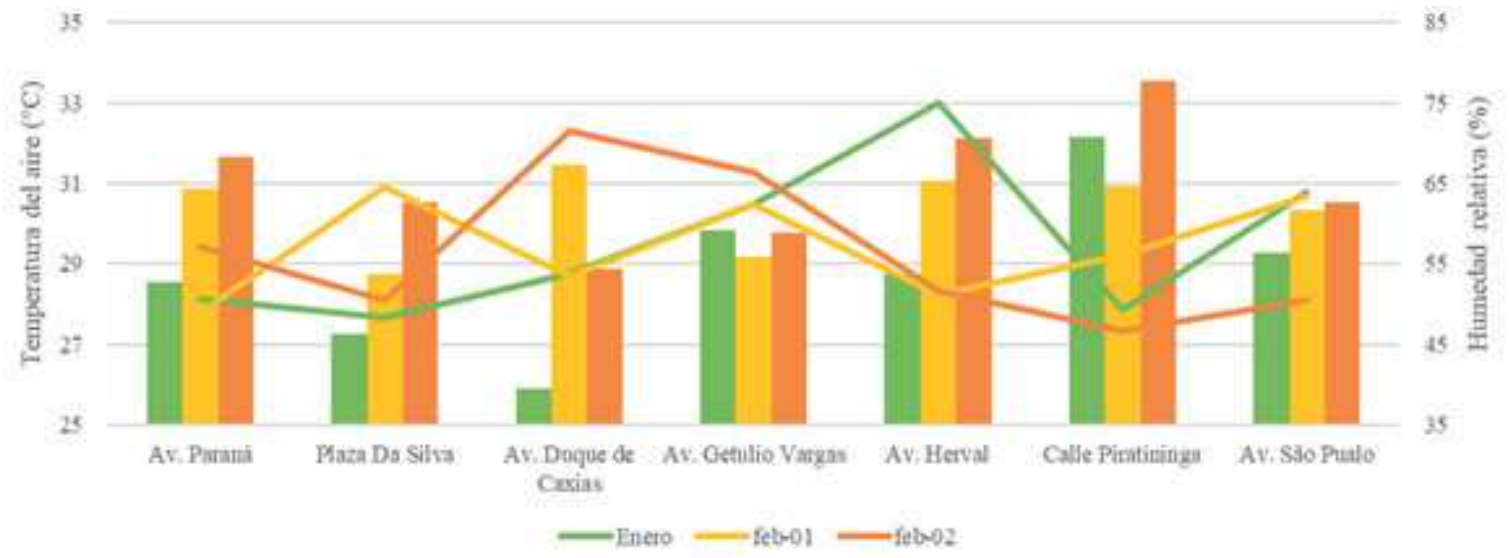

FIGURA 5 - Temperaturas de aire (líneas) y humedades relativas promedias (barras) del periodo de verano. 
superiores o iguales a $80 \%$, esto debido a aguaceros dispersos registrados en la ciudad. La velocidad de los vientos registrados en este periodo estuvo entre $0 \mathrm{~m} / \mathrm{s}$ a $7,29 \mathrm{~m} / \mathrm{s}$.

Ya para el periodo de invierno, en la Figura 6 se presentan las variables climáticas temperatura del aire y humedad relativa. Igual que el periodo de invierno, las mediciones se subdividieron en Junio 1 (primeros 7 días de mediciones levantadas en el mes de Junio), Junio 2 y Julio 1. Las temperaturas promedias del aire estuvieron entre $\operatorname{los} 14,6^{\circ} \mathrm{C}$ y $27,6^{\circ} \mathrm{C}$ (líneas), apenas en dos días fueron medidos valores de temperaturas inferiores a $10^{\circ} \mathrm{C}$, estos dos días pueden ser identificados en los picos de la Figura 7, en el local ubicado en la Intersección de Av. Brasil con Duque de Caxias, en las semanas Junio 1 y Julio. En cuanto a los valores de humedad relativa promedio, estas estuvieron entorno a $\operatorname{los} 25,1 \%$ y $81,0 \%$ (barras), en la primera semana de Junio, específicamente en el local ubicado en la intersección de la Av. Brasil con Av. Getulio Vargas, se registró $17,6 \%$ de humedad relativa, el valor mínimo del periodo, mientras que, en la semana Junio 2, específicamente en el local ubicado en la intersección de la Av. Brasil con la calle Piratininga, se registró el valor máximo de la humedad para el periodo $(93,1 \%)$, esto también debido a los aguaceros dispersos de la temporada. La velocidad de los vientos estuvo entre los $0 \mathrm{~m} / \mathrm{s}$ y $5,86 \mathrm{~m} / \mathrm{s}$.

\section{Análisis de la sensación térmica real versus el Índice PET}

En la Figura 7 se presenta la relación de los votos de sensación térmica real y los valores determinados por el índice PET. Anteriormente, Maringá fue definida como una ciudad subtropical con inviernos leves, claramente esto puede ser visto en la figura anterior. Esta premisa contribuyo para que los votos es estrés para el frio sean pocos, así como, los predictivos por el índice PET. Para el periodo de invierno, el índice considera un gran

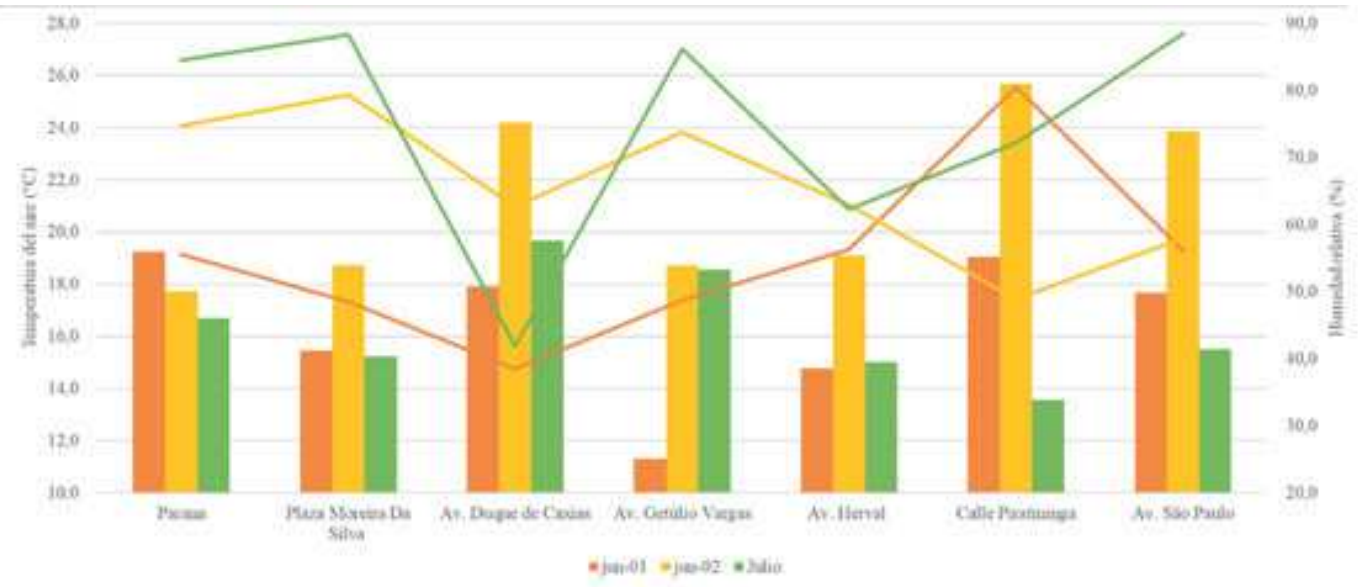

FIGURA 6 - Temperaturas del aire (líneas) y humedades relativas promedias (barras) del periodo de invierno. FUENTE: Autores, 2017. 


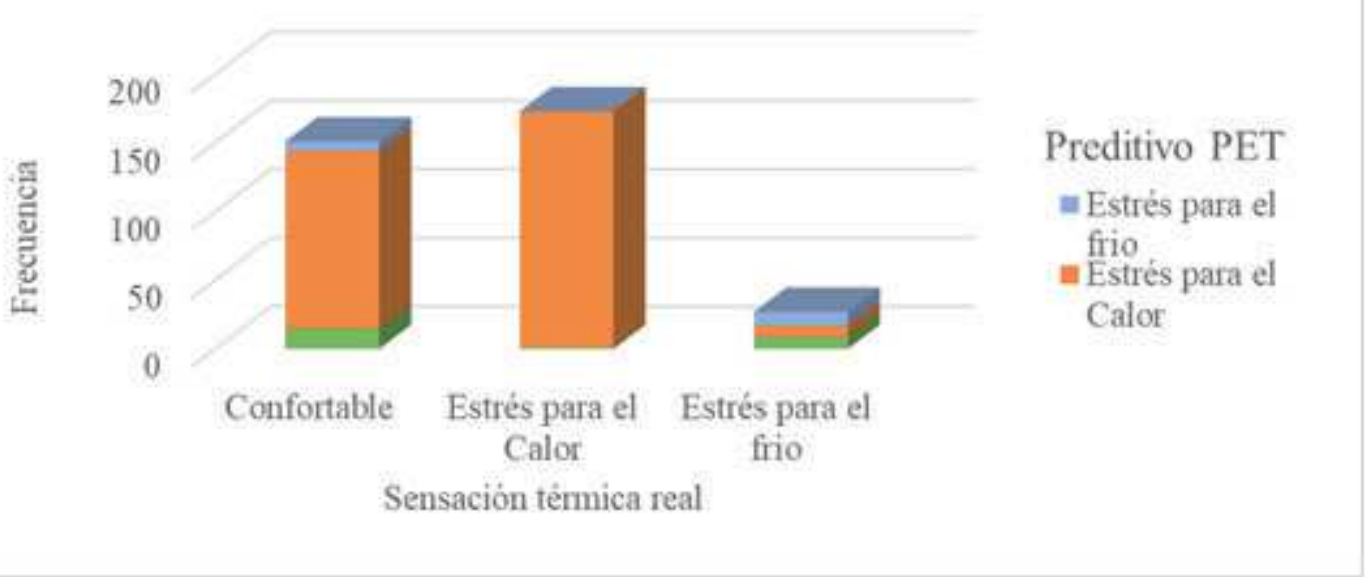

FIGURA 7 - Comparación de los votos de sensación térmica real y los valores del índice PET.

FUENTE: Autores, 2017.

número de personas con estrés para el calor, aunque ellos afirmasen sentir frío, también para el grupo de personas que afirmaron estar confortables, el índice posiciona a varias personas con estrés para el calor, por lo que era de esperar que el índice responda satisfactoriamente con relación a las personas que afirmaron encontrarse con estrés térmico a causa del calor. Esto evidencia claramente la necesidad de que el índice PET sea calibrado para ser utilizados en ciudades cálidas a calurosas y específicamente en la ciudad de Maringá.

Al analizar el porcentaje de acierto y de correlación índice con los votos de sensación térmica real (Tabla 5) se observa que el índice PET tiene

TABLA 5 - Porcentajes de aciertos y correlación del índice PET - Sin Calibración Índice PET $\left({ }^{\circ} \mathrm{C}\right)$

\begin{tabular}{cc}
\hline Porcentaje de aciertos & $\mathbf{5 6 \%}$ \\
\hline Correlación de Pearson & 0,11562109 \\
\hline
\end{tabular}

FUENTE: Autores, 2017. un $56 \%$ de aciertos para la ciudad de Maringá, de los cuales 4,3\% (15 personas) corresponde a las personas que afirmaron estar confortables y que el índice satisface esta condición. Hablando de la correlación, esta también es baja, entre tanto, demuestra una correlación lineal.

Para realizar la calibración del índice fue utilizado el método interactivo variando los límites de cada intervalo interpretativo para maximizar la representatividad de los votos de sensación térmica real de los entrevistados. En total, fueron analizados 3 alternativas y escogida aquella que obtuvo mejor porcentaje de acierto y correlación. De esta forma, en la Tabla 6 se presenta la comparación de los intervalos establecidos por el índice y los propuesto por esta investigación.

Del análisis anterior, es posible identificar los siguientes intervalos de confort y estrés Térmico:

(a) Estrés para el frío, $\mathrm{Ta} \leq 19,5^{\circ} \mathrm{C}$; 
(b) Confort, $19,5^{\circ} \mathrm{C}<\mathrm{Ta}<30^{\circ} \mathrm{C}$; y

(c) Estrés para el calor, $\mathrm{Ta} \geq 30^{\circ} \mathrm{C}$.

Estos intervalos de confort e estrés proporcionaron el porcentaje de aciertos presentado en la Tabla 7, también en esta tabla, se presenta la correlación obtenida con los niveles de confort propuesto y presentados en la tabla anterior. Con la calibración se logró aumentar aproximadamente en $10 \%$ el porcentaje de aciertos, también fue aumentado la correlación entre los votos reales de sensación térmica y las predicciones por el índice, sin embargo este valor todavía es considerado bajo.
Buscando validar los valores obtenidos, estos fueron comparados con los resultados de otras investigaciones que calibraron del índice PET para otras ciudades de Brasil. En la Tabla 8 se presenta esta comparación. El intervalo de confort propuesto en este trabajo es cercano de los valores de la ciudad de Campinas obtenidos en la investigación de Labaki et al. (2012). Analizando los climas característicos de estas ciudades, de acuerdo con la clasificación de Köppen, el clima de Campinas (lat. $22,53^{\circ} \mathrm{S}$, largo $47,04^{\circ} \mathrm{W}$ ) es del tipo Cwa. Tanto para el clima de Maringá, Cfa, y Campinas, Cwa, el clima está caracterizado como templado húmedo

TABLA 6 - Intervalos de nivel de estrés térmico calibrados.

\begin{tabular}{|c|c|c|}
\hline \multirow[t]{2}{*}{ Nivel de estrés térmico } & \multicolumn{2}{|c|}{$\operatorname{PET}\left({ }^{\circ} \mathrm{C}\right)$} \\
\hline & Sin Calibración & Calibración Propuesta \\
\hline Extremo estrés para frio & $<4$ & $<8$ \\
\hline Moderado estrés para frio & $8-13$ & $13-18$ \\
\hline Leve estrés para frio & $13-18$ & $18-19,5$ \\
\hline Leve estrés para calor & $23-29$ & $30-32,5$ \\
\hline Moderado estrés para calor & $29-35$ & $32,5-35$ \\
\hline Fuerte estrés para calor & $35-41$ & $35-38$ \\
\hline Extremo estrés para calor & $>41$ & $38<$ \\
\hline
\end{tabular}

FUENTE: Autores, 2017. 
TABLA 7 - Porcentajes de aciertos y correlación del índice PET-Calibrado Calibración Propuesta - PET $\left({ }^{\circ} \mathrm{C}\right)$.

\begin{tabular}{ll}
\hline Porcentaje de aciertos & $\mathbf{6 7 \%}$ \\
\hline Correlación de Pearson & 0,291690458 \\
\hline
\end{tabular}

FUENTE: Autores, 2017.

con verano caliente, la diferencia entre ellos es en la estación seca, que, para Cwa es en los meses de invierno y para $\mathrm{Cfa}$, no se encuentra indefinida. En cuanto a los porcentajes de aciertos, el valor obtenido en esta investigación es semejante a los porcentajes obtenidos en las demás investigaciones, por tanto, los resultados satisfacen los requerimientos para predecir la sensación de confort y estrés térmico de la ciudad de Maringá.

\section{Conclusión}

A partir de los datos monitoreados en las campañas de campo dentro de la ciudad de Maringá, en el período de verano (Enero y Febrero) e invierno
(Junio y Julio) de 2016, fue posible calibrar el índice PET para la realidad de la ciudad aumentando la predicción de grados de confort y térmico. Analizando los datos climáticos personales y las respuestas de sensación térmica fue posible identificar los siguientes intervalos de confort y estrés térmico, para un porcentaje de aciertos del $67 \%$,

(a) Estrés térmico para frio, $\mathrm{Ta} \leq 19,5^{\circ} \mathrm{C}$;

(b) confort, $29,5^{\circ} \mathrm{C}<\mathrm{Ta}<30^{\circ} \mathrm{C}$; e

(c) Estrés térmico para calor, $\mathrm{Ta} \geq 30^{\circ} \mathrm{C}$.

Posteriormente estos datos fueron comparados con resultados obtenidos en otras investigaciones realizadas en Brasil, de este análisis se demostró que tanto los intervalos de confort y estrés térmico como los valores de porcentajes de aciertos y correlación por más que sean bajos, estos se encuentran dentro de los valores obtenidos en la región.

Finalmente, los resultados de este trabajo pueden ser utilizados como índice predictivo de la sensación térmica de la ciudad de Maringá.

TABLA 8 - Comparación entre investigaciones realizadas dentro de Brasil.

\begin{tabular}{|c|c|c|c|}
\hline \multirow{2}{*}{ Referencia } & \multirow{2}{*}{ Ciudad } & \multicolumn{2}{|c|}{ PET $^{\circ} \mathrm{C}$} \\
\hline & & $\%$ de aciertos & Límite de Confort térmico \\
\hline Monteiro (2008) & San Paulo - SP & 79 & $18-26$ \\
\hline Labaki et al (2012) & Bauru - SP & & $20-29$ \\
\hline Souza (2010) & Salvador - BA & & $26-29$ \\
\hline Hiroshima (2014) & Belo Horizonte $-\mathrm{MG}$ & 71,7 & $23-31$ \\
\hline Autor (2017) & Maringá - PR & 67 & $19,5-30$ \\
\hline
\end{tabular}

FUENTE: Autores, 2017. 


\section{Referencias}

Abreu-Harbich, L. V.; Labaki, L. C.; Matzarakis, A. Thermal bioclimate in idealized urban street canyons in Campinas, Brazil. Theoretical and applied climatology, 115, 333-340, 2014.

Andrade, C. R. M.; Souza, F. C. A cidade de Maringá, PR. O plano inicial e as "requalificações urbanas". Scripta Nova. Revista Electrónica de Geografía y Ciencias Sociales, 12, 2008. Disponivel em: http://www.ub.edu/geocrit/-xcol/55. htm

Araújo, R. R.; Rangel, M. E. S. Crescimento urbano e variações térmicas em São Luis-MA. Revista Geonorte, 3(9), 308-318, 2016.

ASHRAE - American Society of Heating, Refrigeration and Air-Conditioning Engineers. Standard 55-2004 Thermal environmental conditions for human occupancy. Atlanta: ASHRAE, 2004.

Barbirato, G. M.; Souza, L. C. L.; Torres, S. C. Clima e cidade: a abordagem climática como subsídio para estudos urbanos. Maceió: EDUFAL,2007.

Bröde, P.; Krüger, E. L.; Rossi, F. A.; Fiala, D. Predicting urban outdoor thermal comfort by the Universal Thermal Climate Index UTCI--a case study in Southern Brazil. International Journal of Biometeorology, 56(3), 47, 2011, doi: 10.1007/s00484-011-0452-3

Capelli, D. S. A.; Piccolo, M. C.; Campo D. F. A. El clima urbano de Bahía Blanca. Buenos Aires, Dunken, 2005.

Cohen, P.; Potchter, O.; Matzarakis, A. Human thermal perception of Coastal Mediterranean outdoor urban environments. Applied Geography, 37, 1-10, 2013. doi: 10.1016/j. apgeog.2012.11.001

Dacanal, C.; Labaki, L. Ch.; Leite, T. M. S. Vamos passear na floresta! O conforto térmico em fragmentos florestais urbanos. Ambiente Construido, 10(2), 115-132, 2010. Disponível em: http://repositorio.unicamp.br/handle/REPOSIP $/ 35347$

Do Couto N. P. C.; De Musis, C. R.; Biudes, M. S.; Nogueira, J. S.; Nogueira, M. C. D. J. A. Usos dos Índices
PET e UTCI na avaliação do conforto termal no campus da UFMT em Cuiabá-MT, Revista Eletrônica em Gestão, Educação e Tecnologia Ambiental, 9, 2026-2036, 2013. doi: $10.5902 / 223611707707$

Farr, D. Urbanismo sustentável: desenho urbano com a natureza/Douglas Farr; tradução: Alexandre Salvaterra. Porto Alegre: Bookman, 2013.

Hiroshima, S. Q. D. S. Percepção sonora e térmica e avaliação de conforto em espaços urbanos abertos do município de Belo Horizonte-MG, Brazil. São Paulo, Brasil, São Paulo. Tese (Doutorado em Arquitetura e Urbanismo) - USP, 2014.

Höppe, P. The physiological equivalent temperature-a universal index for the biometeorological assessment of the thermal environment. International journal of Biometeorology, 43(2), 71-75, 1999. doi: 10.1007/s004840050118

IAPAR - Instituto Agronômo do Paraná. Cartas climáticas do Paraná: Classificação climática, 2016. Disponível em: http://www.iapar.br/modules/conteudo/conteudo.php?conteudo $=863$. Acesso em: jun. 2017

International Organization for Standardization. ISO 10551. Ergonomics of the thermal environments - Assessment of the influence of the thermal environment using subjective judgment scales. Genève: ISO, 1995.

International Organization for Standardization. ISO 7726. Ergonomics of the thermal environments - Instruments for measuring physical quantities. Genève: ISO, 2006.

IPARDES - Instituto Paranaense de Desenvolvimento Econômico e Social. Caderno Estatístico: Município de Maringá,2016. Disponível em: www.ipardes.gov.br. Acesso em: mar.2016

Krüger, E. L.; Rossi, F. A. Effect of personal and microclimatic variables on observed thermal sensation from a field study in southern Brazil. Building and environment, 46(3), 690-697, 2011. doi: 10.1016/j.buildenv.2010.09.013

Labaki, L. C.; Fontes, M. S. G C.; Bueno-Bartholomei, C. L.; Dacanal, C. Conforto térmico em espaços públicos de passagem: estudos em ruas de pedestres no estado de São Paulo. Ambiente Construído, 12(1), 167-183, 2012. Disponível em: http://www.scielo.br/pdf/ac/v12n1/v12n1a03

Lin, T. P.; Tsai, K. T.; Liao, C. C.; Huang, Y. C. Effects of 
thermal comfort and adaptation on park attendance regarding different shading levels and activity types. Building and Environment, 59, 599-611, 2013. doi: 10.1016/j.buildenv.2012.10.005

Mayer, H.; Höppe, P. Thermal confort of man in different urba environments. Theoretical and applied climatology. Theoretical and applied climatology, 38(1), 43-49, 1987. doi: 10.1007/BF00866252

Monteiro, L. M. Modelos Preditivos de Conforto Térmico: quantificação de relações entre variáveis microclimáticas e de sensação térmica para avaliação e projeto de espaços abertos. São Paulo, Brasil, São Paulo, Tese (Doutorado em tecnologia da Arquitetura e Urbanismo) -USP, 2008.

Monteiro, L. M.; Alucci, M. P. Conforto Térmico em Espaços Abertos com Diferentes Abrangências Microclimáticas: parte 2: proposição de calibração de modelos preditivos. In: Encontro Nacional, 9, Encontro Latino Americano de Conforto no Ambiente Construído. Ouro Preto. 2007, 1231-1240.

Naciones Unidas. Directrices provisionales sobre clasificaciones internacionales uniformes de edades, SER.M/74, 1983. Disponible en: https://unstats.un.org/unsd/publication/SeriesM/SeriesM_74s.pdf. Acceso en: mar. 2016
Pezzuto, C. C. Avaliação do ambiente térmico nos espaços urbanos abertos. Estudo de caso em São Paulo, Brasil, Campinas. Tese (Doutorado em Engenharia Civil) - UEC, 2007. p. 197.

Rego, R. L. O desenho urbano de Maringá e a ideia de cidade-jardim. Acta Scientiarum. Technology, 23(6), 1569-1577, 2001. doi: 10.4025/actascitechnol.v23i0.2801

Rossi, F. A. Proposição de metodologia e de modelo preditivo para avaliação da sensação térmica em espaços abertos em Curitiba. Paraná, Brasil, Curitiba. Tese (Doutorado em Tecnologia) - UTFP, 2012.

Sartori, M. D. G. B. Clima e Percepção. São Paulo, Brasil, São Paulo, Tese (Doutorado em Geografia Física). USP, 2000 .

Souza, S. H. M. D. Avaliação do desempenho térmico nos microclimas das praças: Piedade e Visconde de Cayrú, Salvador/BA. Bahia, Brasil, Salvador. Tese (Mestrado em Engenharia Ambiental Urbana) - UFB, 2010. 